\title{
Reliability assessment of ductile shells
}

\author{
L. M. C. Simões*
}

\begin{abstract}
This paper concerns the development of mathematical programming techniques for the reliability assessment of three-dimensional structures, assuming an ideal rigid-plastic behaviour of the material. Linear approximations of the kinematic compatibility relations are formulated and this requires the assumption of parametric stress and displacement fields, which are constructed by means of finite element procedures. According to the first-order second-moment method, the identification of the reliability index is then formulated as a constrained concave quadratic minimization. Numerical results for a shallow shell model are presented.
\end{abstract}

Keywords: optimization, plasticity structures, reliability, shells

\section{Introduction}

The discrete nature of mathematical programming (MP) implies that continuum structures must be treated in a discretized manner. The literature on finite element method (FE) formulation of plastic limit shell problems is very limited. A formulation for the plastic limit analysis of cylindrical shelis was developed by Kalanta and Cyras $^{1}$, and Hung and Ramsart ${ }^{2}$, whilst a formulation for shallow shells was presented by Cyras et al. ${ }^{3}$ and Küfner and Lee ${ }^{4}$. The formulation of the problem of the plastic limit analysis of plates and shells by finite elements has been presented by Da Fonseca ${ }^{5}$ where a linearized yield condition is used, the problem is reduced to a pair of primal-dual linear programs (LP). The optimal solution to the discretized problem may provide a bound to the solution of the continuous problem, but the existence and nature of such a bound depends on the form of the finite element modelling. Thus, where statical admissibility is imposed, the FE/LP solution provides a lower bound to the collapse load parameter for the continuous plate. Similarly, if kinematic admissibility is imposed, then the FE/LP solution provides an upper bound to that parameter. Plastic limit analysis yields the collapse load which does not necessarily lead to the stochastically most relevant event. Moreover the contribution of other stochastically important mechanisms must be taken into account. Assessment of the reliability of a structure has to take into account that during its design life the structure is generally subjected to a number of varying loads and their combinations and that its resistance may deteriorate with time. In this context the problem arises as to how to evaluate the conditional failure probability of the shell given a certain load event. In order to avoid the difficult numerical integration of the probability distribution functions involved, the firstorder second-moment approximation is employed. The reliability index is obtained from the limit-state equation by solving a nonconvex fractional programming problem. This MP can be converted in the minimization of a concave quadratic function over a linear domain, which is also nonconvex. A branch and bound technique is used

* Departamento de Engenharia Civil, Faculdade de Ciencias e Tecnologia Universidade de Coimbra, 3049 Coimbra, Portugal to solve this MP and to enumerate the remaining local solutions which may also be important for the reliability assessment.

\section{Problem formulation}

\section{Assumptions}

The finite element method was chosen because of its versatility in the automatic generation of the stochastically most relevant failure mechanism; that is, the mechanism with the smallest reliability index $\beta$ and the highest probability of failure $\mathrm{P}_{\mathrm{F}}$. Plastic collapse is the only possible failure mode. The kinematics and statics of ductile shallow shells are governed by relations where the stretching is dissociated from the bending problem. The kinematic model under consideration can be taken as the superimposition of stretching and bending models and the field functions required may be obtained through the superimposition of the field functions defined for those models. The mathematical characterization of the plastic behaviour of a general shell would require a yield criterion involving 10 stress-resultants and 10 strain-resultants. However, as for flexural plates, the effect of the two transverse shear forces is generally negligible. Also, since every finite element is shallow, the planar shear forces and the twisting moments are regarded as equal. Since the direct integration of the yield function across the curved plate thickness is beset with difficulties, the yield criterion used here considers separately the stretching and bending problems with no interaction between them. The limit-state equation is defined by the yield conditions at the control nodes. If a yield surface is described by a single random variable, it has a deterministic shape with a random size. Without any complications (besides the increase in problem size), a random shape of each yield surface can also be modelled by associating a random resistance variable to each face of the surface. The magnitudes of static loads, $L_{1}, L_{2}, \ldots, L_{\mathrm{r}}$ which form the load vector $L$ are random but their positions are deterministic. The magnitudes of stress capacities, $\sigma_{1}, \sigma_{2}, \ldots, \sigma_{5}$ are random variables which make up vector $\sigma$. The statistical dependence between any pair of loads $L_{1}, L_{\mathrm{m}}$ and any pair of stress capacities $\sigma_{j}, \sigma_{k}$ is accounted for through the coefficients of correlation $\rho\left(L_{1}, L_{\mathrm{m}}\right)$ and $\rho\left(\sigma_{\mathrm{j}}, \sigma_{\mathrm{k}}\right)$, 
respectively. Consistent with a first-order second-moment reliability approach, the minimum statistical information required for the evaluation of the optimum solution is:

(1) The mean values of the loads $\mu_{L_{1}}, \mu_{L_{2}}, \ldots, \mu_{L_{\mathrm{r}}}$ ? which make up the vector $\mu_{L}$, the coefficients of variation of the loads $\Omega\left(L_{1}\right), \Omega\left(L_{2}\right), \ldots, \Omega\left(L_{\mathrm{r}}\right)$ which make up the vector $\Omega_{L}$, and the coefficients of correlation between pairs of loads which form a square symmetrical correlation matrix denoted by $\mathrm{C}_{\mathrm{u}}$.

(2) The mean values of the stress capacities which make up the vector $\mu_{\mathrm{s}}$, the coefficients of variation of the stresses $\Omega\left(\sigma_{1}\right), \Omega\left(\sigma_{2}\right), \ldots, \Omega\left(\sigma_{s}\right)$, which make up the vector $\Omega_{\sigma}$ and the coefficients of correlation between pairs of plastic moments which form a square symmetrical matrix denoted by $\mathrm{C}_{\mathrm{g}}$.

\section{Kinematic relations}

The formulation of the FEM has evolved from a purely physical approach in which the FE level relations were directly derived from the infinitesimal element level relations, to the presently preferred approach based on variational principles. It will be recalled that early development of direct methods led to inconsistent formulations which were subsequently connected by using variational procedures. In order to overcome the lack of governing variational principles in certain problems, the FEM can also be regarded as the method of weighted residuals (MWR) applied to each individual FE, where consistency is ensured by the Galerkin criterion. In the formulation of the finite element method, three distinct levels (i) the infinitesimal element level, (ii) the finite element level and (iii) the structural level are defined. The starting point for the kinematic relations are the infinitesimal element level strain-resultant/displacement relations:

$$
\varepsilon-\mathrm{D} u=0
$$

where $\mathrm{D}$ is the matrix differential operator and the kinematic variables $\varepsilon$ and $u$ are activated at the instant of structural collapse.

The finite element level relations may now be obtained, for which approximations may have to be introduced. If, as a consequence, the kinematic relations are no longer satisfied locally, they can be written

$$
\varepsilon-\mathbf{D} u=\delta(\varepsilon)
$$

where the net strain-resultants $\delta(\varepsilon)$ are zero if the strainresultants $\varepsilon$ are directly derived from the displacement field $u$, in which case strain compatibility is automatically ensured. The finite element method implies the definition of the distribution of the independent variables inside each finite element in terms of nodal values. Continuous fields are assumed inside every finite element, but lack of continuity may be allowed to develop across the interelement boundaries. It must be clear that the nodal values are not necessarily the values of the variables at the nodes. For example, a true displacement $u$ may be defined by nodal values that can be rotations, curvatures or any other derivatives of the displacements. In the present case, the static $\sigma$ and kinematic $u$ independent variables are expressed:

$$
\begin{aligned}
& \sigma=\Gamma_{\sigma} \sigma^{e} \\
& u=\Gamma_{u} u^{a}
\end{aligned}
$$

respectively, in terms of static nodal values at $e$ nodes and kinematic nodal values at $a$ nodes, resulting for Equation 2:

$$
\varepsilon-\mathrm{D}_{u} u^{a}=\delta(\varepsilon)
$$

The use of an approximating function $\Gamma_{\sigma}$ signifies that inter-element equilibrium is no longer necessarily satisfied, but the approximating function $\Gamma_{u}$ in Equation 4 does not imply incompatibility of strains, provided that the latter are now defined as functions of the new variables $u^{a}$. Equation 4 is then suitably pre-multiplied and integrated throughout the entire finite element,

$$
\int_{\mathrm{V}} \Gamma_{\sigma}^{\mathrm{l}} \varepsilon \mathrm{dV}-\int_{\mathrm{V}} \Gamma_{\sigma}^{\mathrm{t}} \mathrm{D} \Gamma_{\mathrm{u}} u^{a} \mathrm{dV}=\int_{\mathrm{V}} \Gamma_{\sigma}^{\mathrm{t}} \delta(\varepsilon) \mathrm{dV}
$$

An approximation is now introduced into the kinematic relations. If the finite element volume is subdivided into $\mathrm{c}$ subdomains $\mathrm{V}_{\mathrm{j}}(\mathrm{j}=\mathrm{I}, \ldots, \mathrm{c})$, each associated with a control point $\mathrm{c}_{\mathrm{j}}$ where matrix $\Gamma_{g}^{\mathrm{t}}$ assumes the value $\left(\Gamma_{\sigma}^{\mathrm{t}}\right)_{j}$ and the strain-resultants $\varepsilon$ are regarded as having a constant value $\varepsilon_{j}$ inside each volume $V_{j}$, the following approximation may be made:

$$
\int_{V} \Gamma_{\sigma}^{t} \varepsilon d V=\sum_{j=1, c} \int_{V_{j}} \Gamma_{\sigma} \varepsilon d V_{j} \cong \sum_{j=1, c}\left(\Gamma_{\sigma}^{t}\right)_{j} \varepsilon_{j} V_{j}
$$

The nodes $\mathrm{c}$ are generally the control nodes selected to enforce the constitutive relations throughout the finite element. Defining the total nodal strains,

$$
g_{j}=\int_{v_{j}} \Delta \varepsilon d V
$$

this leads to the following result:

$$
\int_{V} \Gamma_{\sigma}^{\mathrm{t}} \Delta \varepsilon \mathrm{dV} \cong\left(\Gamma_{a}^{\mathrm{e}}\right)^{\mathrm{t}} \mathrm{g}^{\mathrm{e}}
$$

and Equation 5 may be written in the following form:

$$
\left(\Gamma_{\sigma}^{\mathrm{e}}\right)^{t} \mathrm{~g}^{\mathrm{c}}-\int_{\mathrm{V}} \Gamma_{\sigma}^{\mathrm{t}} \mathrm{D} \Gamma_{u} \mathrm{dV} u^{a}=\int_{\mathrm{V}} \Gamma_{\sigma}^{\mathrm{t}} \delta(\varepsilon) \mathrm{dV}
$$

This approximation signifies that the strain-resultants $\varepsilon$ field is assumed to be such that condition (1) is no longer necessarily satisfied everywhere and, consequently, quantities $\delta(\varepsilon)$ may be non-zero. It is concluded that this formulation allows both the equilibrium and the compatibility to be locally contravened. Nevertheless, a global compatibility is enforced at finite element level by setting to zero the right-hand side terms in Equation 9. The finite element kinematic relations may be written in a more compact form:

$$
\left(\Gamma_{\sigma}^{\mathfrak{c}}\right)^{\mathrm{c}} \mathrm{g}^{\mathrm{c}}-\mathrm{E} u^{a}=0
$$

where

$$
\mathbf{E}=\int_{\mathrm{V}} \Gamma_{\sigma}^{\mathrm{t}} \mathrm{DF}_{u} \mathrm{dV}
$$

The static and kinematic nodal variables are transformed from the local system of reference to the global according to,

$$
\sigma^{e}=\mathrm{T}_{\sigma}^{e} \sigma^{e} ; \quad \sigma^{e}=\left(\mathrm{T}_{\sigma}^{e}\right)^{t} \sigma^{e}
$$




$$
\begin{aligned}
& u^{a}=\mathrm{T}_{u}^{a} u^{a} ; \quad u^{a}=\left(\mathrm{T}_{u}^{a}\right)^{t} u^{a} \\
& \mathrm{~g}^{\mathrm{c}}=\mathrm{T}_{\mathrm{g}}^{\mathrm{c}} g^{\mathrm{c}} ;-g^{\mathrm{c}}=\left(\mathrm{T}_{\mathrm{g}}^{\mathrm{c}}\right)^{\mathrm{t}} g^{\mathrm{c}}
\end{aligned}
$$

The kinematic relations at finite element level in global coordinates become

$$
\left(\mathbf{T}_{\sigma}^{e}\right)^{t}\left(\Gamma_{\sigma}^{c}\right)^{t} \mathrm{~T}_{g}^{c} g^{c}-\left(\mathrm{T}_{\sigma}^{e}\right)^{t} E \mathrm{~T}_{u}^{a} u^{a}=0
$$

or in a more compact form

$$
\mathrm{R} g^{\mathrm{c}}-E u^{a}=0
$$

If nodes $e$ are also control nodes $\mathrm{c}, \Gamma_{\sigma}^{\mathrm{c}}$ is a unit matrix, matrices $\mathrm{T}_{\sigma}^{\mathrm{e}}$ and $\mathbf{T}_{\mathrm{g}}^{\mathrm{c}}$ express orthogonal transformations laws for vectorially equivalent variables and,

$$
\mathrm{T}_{\sigma}^{\mathrm{c}}=\mathrm{T}_{\mathrm{g}}^{\mathrm{c}}
$$

If a similar procedure is adopted for the entire system of finite elements, the structural level compatibility relations can be written as:

$$
\mathrm{R}^{\mathrm{s}} \mathrm{g}^{\mathrm{ss}}-E^{\mathrm{s}} u^{a \mathrm{~s}}=0
$$

If all shared control nodes are also nodes $c$, the yield conditions can be stated identically for all cs control nodes and matrix $\mathrm{T}_{\mathrm{g}}^{\mathrm{c}}$ becomes a unit matrix.

\section{Plasticity relations}

For the purpose of problems involving rigid-plastic behaviour, only the plastic phase of the structural material behaviour has to be characterized. Yield conditions on the stresses, the conditions for the plastic strains to take place, the flow rule and plastic dissipation energy must all be postulated. The conditions for yielding to occur may be defined through an inequality involving a function (generally non-linear) of the stress-state, as follows:

$$
\pi(\sigma) \leqslant 0
$$

According to plasticity theory, the stress components $\sigma$ and the correspondent strain components $\varepsilon$ are related during collapse as follows:

$$
\varepsilon=\frac{\partial \pi}{\partial \sigma} c * \quad \begin{array}{ll}
\varepsilon * \geqslant 0 & \text { if } \pi(\sigma)=0 \\
\varepsilon *=0 & \text { if } \pi(\sigma)<0
\end{array}
$$

The yield conditions require the stress components to be transformed from the global to the local system of reference, thus allowing for the stress field function which is defined in local coordinates. If such a hypersurface is approximately replaced by a set of hyperplanes, the corresponding linearized yield conditions for every control node $\mathrm{c}_{\mathrm{j}}$ are:

$$
\pi *_{j}=Q_{j}^{i} \sigma_{j}-\sigma *_{j} \leqslant 0
$$

The correct characterization of plastic behaviour cannot generally be achieved everywhere in the finite element because the discretization of the problem also requires the plastic relations to be stated at a finite number of control nodes and it may then be impossible to guarantee the satisfaction of all plastic conditions in:ile the element. Special techniques may be employed to determine the convenient locations of the control nodes, but the problem is always to state the infinitesimal element level plastic relations at the selected control nodes. In matrix notation

$$
\pi *=\mathrm{Q}^{\mathrm{t}} \sigma-\sigma *=\left[\mathrm{Q}^{\mathrm{l}} \mathrm{T}_{\sigma}^{\mathrm{t}}\right] \sigma-\sigma * \leqslant 0
$$

or introducing the stress field function

$$
\pi *=\left[\mathrm{Q}^{\mathrm{T}} \mathrm{T}_{\sigma}^{\mathrm{T}} \Gamma_{\sigma}\right] \sigma^{e}-\sigma * \leqslant 0
$$

and returning to the stress global components by means of the transformation:

$$
\pi *=\left[\mathrm{Q}^{\prime} \mathrm{T}_{\sigma}^{\mathrm{l}} \Gamma_{\sigma} \mathrm{T}_{\sigma}^{e}\right] \sigma^{e}-\sigma * \leqslant 0
$$

If these conditions are stated for the control nodes,

$$
\pi_{*}^{\mathrm{c}}=\left[\left(\mathrm{Q}^{\mathrm{c}}\right)^{\mathrm{t}}\left(\mathrm{T}_{\sigma}^{\mathrm{e}}\right)^{\mathrm{t}} \Gamma_{\sigma}^{\mathrm{c}} \mathrm{T}_{\sigma}^{e}\right] \sigma^{e}-\sigma_{*}^{\mathrm{c}}\left[\mathrm{R}\left(\mathrm{Q}^{\mathrm{c}}\right)\right]^{\mathrm{t}} \sigma^{e}-\sigma_{*}^{\mathrm{c}} \leqslant 0
$$

or, for all cs nodes their assemblage can be written as,

$$
\pi_{*}^{\mathrm{cs}}=\left[\mathrm{R}^{\mathrm{s}}\left(\mathrm{Q}^{\mathrm{cs}}\right)\right]^{\mathrm{i}} \sigma^{\mathrm{e}}-\sigma_{*}^{\mathrm{cs}} \leqslant 0
$$

For a stable material in the Drucker sense, the yield surface is convex and the kinematic variables are defined by an associated flow rule. The stress field generated throughout the structure should not contravene the yield conditions at any point in the structure, but this is only easily achieved for a constant or a linear stress field. In any case, the yield conditions are enforced directly only at a selected set of control points $\mathrm{c}$. If the control nodes $\mathrm{c}$ and $e$ are the same, matrix $R$ becomes a unit matrix, and the flow rule for a control node $c_{j}$ is

$$
g_{\mathrm{j}}=\int_{\mathrm{V}_{\mathrm{j}}} \varepsilon_{\mathrm{j}} \mathrm{dV}=\int_{\mathrm{V}_{\mathrm{j}}} \mathrm{Q} \varepsilon_{\mathrm{j}} \mathrm{dV}=\mathrm{Q} g_{* \mathrm{j}}
$$

where $g_{* i} \geqslant 0$ are the total plastic parameters associated with the subdomain of unspecified volume $V_{j}$ inside which the material properties (expressed through matrix Q) are assumed constant. Matrix Notation 25 becomes for all cs control nodes,

$$
g^{\mathrm{cs}}=\mathrm{Q}^{\mathrm{cs}} g_{*}^{\mathrm{cs}}
$$

Now, since plastic deformation is only possible on the yield hypersurface, the parity condition for node $c_{j}$ is transformed as follows:

$$
\int_{\mathrm{V}_{\mathrm{j}}} \pi *_{\mathrm{j}} \varepsilon *_{\mathrm{j}} \mathrm{dV} \cong \pi *_{\mathrm{j}} \int_{\mathrm{V}_{j}} \varepsilon *_{\mathrm{j}} \mathrm{dV}=\pi *_{\mathrm{j}} g *_{\mathrm{j}}
$$

where besides the assumption of constant plastic potential (that is of constant stress field, which was also considered in Equation 6), the assumption of constant strain field is implied. Indeed, that parity condition allows plastic deformations to be generated simultan. eously at any point inside the subdomain of volume $\mathrm{V}_{j}$. Consequently, another approximation is introduced into the kinematics when the strain field is non-constant. The parity condition for all cs nodes may be stated in the following complementary form:

$$
\left(\pi_{*}^{\mathrm{cs}}\right)^{\mathrm{l}} g_{*}^{\mathrm{cs}}=0
$$

Similarly, if the assumption of constant stress field and of constant strain field is introduced, the total plastic dissipation energy for all cs control nodes becomes,

$$
\mathrm{D}^{\mathrm{cS}}=\left(\sigma^{\mathrm{cs}}\right)^{\prime} g^{\mathrm{cs}}=\left(\sigma_{*}^{\mathrm{css}}\right)^{\mathrm{t}} g_{*}^{\mathrm{cs}} \geqslant 0
$$

For the sake of simplicity the bar denoting the global coordinates and the superscripts e, c, a, cs, as, cs will be dropped. 


\section{Reliability assessment}

\section{Collapse of ductile structural systems}

In the case of structures composed of ductile members such as components with elastic-perfectly-plastic behaviour, the structural strength would be independent of the failure sequences of the component. For this class of structural systems, the collapse of a system would be through the formation of plastic mechanisms. In this regard, the performance function for ith plastic mechanism may be defined as,

$$
Z_{\mathrm{i}}=\Sigma_{\mathrm{j}} a_{\mathrm{ij}} \sigma_{\mathrm{j}} *-\Sigma_{\mathrm{k}} b_{\mathrm{ik}} L_{\mathrm{k}}
$$

where $\mathrm{j}$ includes all the areas where yielding occurs in mechanism $i$, whereas $k$ includes all loads active in mechanism i. The collapse of a system through mechanism $i$, therefore, is the event $F_{i}=\left(Z_{i}<0\right)$. Then, if there are $\mathrm{m}$ possible failure mechanisms the system collapse,

$$
\begin{aligned}
\mathrm{P}_{\mathrm{F}}\left(\mathrm{C}_{\mathrm{s}}\right) & =\mathrm{P}\left(\mathrm{Z}_{1}<0 \cup Z_{2}<0 \cup \ldots \cup \mathrm{Z}_{\mathrm{in}}<0\right) \\
& =\mathrm{P}\left(\mathrm{F}_{1} \cup F_{2} \cup \ldots \cup \mathrm{F}_{\mathrm{m}}\right)
\end{aligned}
$$

In general, $Z_{i}$ are correlated random variables; consequently, calculation of the collapse probability, $P_{f}\left(C_{s}\right)$, will require the multiple integration of joint probability distributions of the correlated random variables $Z_{i}, i$ $=1,2, \ldots, \mathrm{m}$. In the first order second moment method the reliability index, $\beta_{i}$, for the performance function $Z_{i}$ is given by:

$$
\beta_{i}=\mu_{Z_{i}} / \sigma_{Z_{i}}
$$

where $\mu_{Z_{i}}$ and $\sigma_{Z_{i}}$ are mean and standard deviation of $Z_{i}$, respectively. As $Z_{i}$ is a linear function of the number of random variables $\sigma *$ and $L$, the distribution of $Z_{i}$ tends to normal (based on central limit theorem) irrespective of the individual distributions of the variables. Hence using normal distribution for $Z_{i}$, the failure probability for mechanism $i, \mathrm{p}_{\mathrm{F}_{i}}$ is given by:

$$
\mathrm{p}_{\mathrm{F}_{i}}=\Phi\left(-\beta_{i}\right)=1-\Phi\left(\beta_{i}\right)
$$

where $\Phi$ is the cumulative probability of a standardized normal variable. Variation of the parameters of the probability distributions, the respective types of distributions and the correlation among the variables involved have quite a significant effect on the results.

\section{Computation of the reliability index}

By associating the finite element strains associated with the stress capacities represented by the same random variable $g_{t}$ through the incidence matrices $J_{g}$ and the displacements of the point loads for in the case of uniformly distributed load, displacements of the equivalent nodal forces) linked by the same random variable $u_{t}$ through $J_{u}$, one has:

$$
g_{t}=J_{g} g ; \quad u_{t}=J_{u} u
$$

For statistically independent random normal variables, the identification of the stochastically most important mechanism consists of finding the position of the limitstate equation closer to the origin of the reduced normal variables. This amounts to minimizing the distance- reliability index $-\beta$ given by (Shinozuka ${ }^{6}$ ):

$$
\min \beta=\frac{\left[\mu_{\sigma}^{2} g_{t}-\mu_{L}^{i} u_{t}\right]}{\sqrt{\left(\sigma_{\sigma}\right)^{2 t}\left(g_{t}\right)^{2}+\left(\sigma_{L}\right)^{2 t}\left(u_{t}\right)^{2}}}
$$

subject to the linear incidence equations (Equation 34), the compatibility relations (Equation 16) and sign constraints on the variables. If matrices $\mathrm{C}_{\mathrm{g}}$ and $\mathrm{C}_{\mathrm{u}}$ represent the correlations between the bending moments of resistance and between the loads, respectively, the reliability index is given by

$$
\beta=\frac{\left[\mu_{\sigma}^{t} g_{t}-\mu_{L}^{\prime} u_{t}\right]}{\sqrt{\left(g_{t}\right)^{t}\left(\sigma_{\sigma}\right)^{t} C_{g}\left(\sigma_{\sigma}\right)\left(g_{t}\right)+\left(u_{t}\right)^{t}\left(\sigma_{L}\right)^{t} C_{u}\left(\sigma_{L}\right)\left(u_{t}\right)}}
$$

For random variables that are correlated, the original variates may be transformed to a set of uncorrelated variables. The required set of uncorrelated transformed variates can be obtained through an orthogonal transformation. If the probability distribution functions of the random variables are not Gaussian, the Rosenblatt? transformation may be used.

These mathematical programs belongs to the class of fractional programming problems (Schaible ${ }^{8}$ ). Since $\left[\mu_{\sigma}^{t} g_{t}-\mu_{L}^{t} u_{2}\right]$ is always positive, (Equation 36) shares its solutions with the minimization of the quadratic concave function,

$$
\begin{aligned}
\min -1 / \beta^{2}= & -\left(g_{t}\right)^{l}\left(\sigma_{\sigma}\right)^{l} C_{g}\left(\sigma_{\sigma}\right)\left(g_{L}\right) \\
& -\left(u_{t}\right)^{t}\left(\sigma_{L}\right)^{t} C_{u}\left(\sigma_{L}\right)\left(u_{t}\right)
\end{aligned}
$$

subject to

$$
\begin{aligned}
& \mu_{\sigma}^{r} g_{t}-\mu_{L}^{t} u_{t}=1 \\
& g_{t}=J_{g} g ; \quad u_{t}=J_{u} u \\
& R g-E u=0 \\
& g \geqslant 0, u \geqslant 0, g_{t} \geqslant 0, u_{t} \geqslant 0
\end{aligned}
$$

that is a quadratic concave minimization. This type of problem cannot be solved by convex programming techniques because of the possibility of nonglobal local minima. The global optimum of these programs gives the plastic deformations for the stochastically most important mechanism and the reduced random variables can be evaluated by

$$
\begin{aligned}
& \sigma^{\prime}=-\sigma_{\sigma} g_{s} \beta^{2} \\
& L^{\prime}=\sigma_{L} u_{t} \beta^{2}
\end{aligned}
$$

\section{Solution of a concave quadratic minimization problem}

The most general methods for global optimization which are appropriate to deal with nonconvexities can be divided in two classes: deterministic and stochastic. Among the latter, the most important approaches for concave quadratic programming are enumerative techniques, cutting-plane methods, branch and bound, bilinear programming methods or different combinations of these techniques. Computational results on most of these methods seem to be limited to cases where the number of nonlinear variables is less than 15 . The few implementable approaches are for functions of special structure such as quadratic or separable concave and employ branch and bound (B\&B) techniques in conjunction 
with underestimating linear (or piecewise linear) problems. The two main ingredients are a combinatorial tree with appropriately defined nodes and some upper and lower bounds to the final solution associated with each node of the tree. It is then possible to eliminate a large number of possible solutions without evaluating thern. As the implicit enumeration program relies on an upper bound, its efficiency can be greatly improved by providing a good feasible initial solution. A partial solution is said to be fathomed if the best feasible completion of the solution can be found. No matter how the design variables are chosen it will be impossible to find a feasible completion of smaller distance than that previously found. If a partial solution is fathomed this means that all possible completions of this partial solution have been implicitly enumerated and therefore need not be explicitly enumerated. When the last node is fathomed the algorithm terminates with the optimum, solution. Backtracking in the tree is performed so that no solution is repeated or omitted from consideration. The algorithms described in Reference 9 are more appropriate to solve concave quadratic minimization of larger systems.

\section{Reduction to separable form}

For simplicity of notation, the concave quadratic program involving correlated random variables can be written in the following form:

$$
\begin{aligned}
& \text { Min } \psi(z, y)=-1 / 2 z^{i} \mathrm{Q} z \\
& \text { over } \Omega=\left\{(z, y): A_{1} z+A_{2} y=b, z \geqslant 0, y \geqslant 0\right\}
\end{aligned}
$$

with $\mathrm{Q}$ a positive definite symmetric matrix, and $\mathrm{A}_{1}$ and $\mathrm{A}_{2}$ having $\mathrm{m}$ rows, $z, y$ are $\mathrm{n}$ and $\mathrm{m}$ vectors corresponding to the random variables and the rotations of the critical sections, respectively. To carry out the reduction to separable form it is necessary to compute the real eigenvalues $\lambda_{1}, \lambda_{2}, \ldots, \lambda_{n}$ of $Q$ and the corresponding eigenvectors $\mathrm{u}_{1}, \mathrm{u}_{2}, \ldots, \mathrm{u}_{\mathrm{n}}$. Then $\mathrm{Q}=\mathrm{UDU}^{\mathrm{t}}$ where $\mathrm{U}=\left[\mathrm{u}_{1}, \ldots, \mathrm{u}_{\mathrm{n}}\right], \mathrm{D}=\operatorname{diag}\left[\lambda_{1}, \ldots \lambda_{\mathrm{n}}\right]$. first

The multiple-cost-row linear program must be solved

$$
\begin{aligned}
& \max u_{i} t_{z} \\
& \operatorname{st}(z, y) \in \Omega, \quad i=1,2, \ldots, \mathrm{n}
\end{aligned}
$$

Denote by $\beta_{i}$ the corresponding optimal values. The concave quadratic programming can be formulated as a separable programming in terms of the new variables $x_{i}$,

$$
\begin{aligned}
& \quad \min \phi(x)=\sum_{i=1, \mathrm{n}}-1 / 2 \lambda_{i} x_{i}^{2} \\
& \qquad \text { st } \mathrm{A}_{3} x+\mathrm{A}_{2} y=b, \quad 0 \leqslant x_{i} \leqslant \beta_{i}, y \geqslant 0 \\
& \text { where } \mathrm{A}_{3}=\mathrm{A}_{1} \mathrm{U} .
\end{aligned}
$$

\section{Linear underestimator}

The smallest rectangular domain $\mathrm{R}_{x}$ in the $x$-space can be constructed by using $\beta_{i}$. The convex envelope of a function over a closed convex set is the highest convex function which everywhere underestimates the function. For a quadratic concave function $-x^{2}$ given in the interval $[0, \beta]$ its convex underestimate is the affine function (linear plus a constant) passing through the endpoints of the given function graph. A linear function $\Gamma(x)$ which interpolates $\phi(x)$ at every vertex of $R_{x}$ and underestimates $\phi(x)$ on $\mathrm{R}_{x}$ is given by,

$$
\Gamma(x)=\sum_{i=1, \mathrm{n}}-1 / 2 \lambda_{i} \beta_{i} x_{i}
$$

and $\mathrm{R}_{x}=\left[x: 0 \leqslant x_{i} \leqslant \beta_{i} ; \quad i=1, \ldots, \mathbf{n}\right\}$

The following linear underestimating program, which differs from the multiple-cost-row only in its objective function, must be solved,

$$
\min \Gamma(x) \text { over }(x, y) \in \Omega
$$

The solution to this problem will give a vertex $v=(x, y)$ which is a candidate for the global minimum $\psi^{*}$ of the original problem.

\section{Outside-in approach}

In the sequel the algorithm originally presented by Falk $^{10}$ for separable functions is outlined. The algorithm first constructs and solves the linear underestimating approximation based on the enclosing rectangle. Considering the quadratic concave program (Equation 40), let $x^{p}$ be the solution of the linear underestimating subproblem (Equation 43), with $p=1$. If the number of nonlinear variables is small, $\Gamma\left(x^{p}\right)=\phi\left(x^{p}\right)$ and $x^{p}$, is the desired global solution. If $\Gamma\left(x^{p}\right)$ does not coincide with $\phi\left(x^{p}\right)$, one partitions the intervals $\left[0, \beta_{i}\right]$ in order to have a tighter objective function. Take as incumbent bound $v$ the objective function of the original problem $\phi\left(x^{p}\right)$. Equation 43 is replaced by a set of problems that bound the original problem in the sense that there exist one optimal solution $x^{*}$ for at least one problem $j \in \mathrm{Wp}$. Supposing an optimal solution to each such problem is obtained and let

$$
x^{s}=\min _{j \in \mathbf{W} p} \Gamma\left(x^{s}\right)=d^{t} x^{j}+k
$$

If $\Gamma\left(x^{s}\right)$ does not coincide with $\phi\left(x^{s}\right)$, one of the problems of the bounding set is replaced by a set of new problems. If $\phi\left(x^{s}\right)$ is lower than the incumbent $\mathrm{v}$, this upper bound will be updated. Make $p=p+1$. The problem $s$ is replaced by a set $\mathrm{W}^{p}$, such that $\mathrm{W}^{p},=\left(\mathrm{W}^{p-1}-\{s\}\right)$ $\cup \mathrm{W}^{s}$ contains an optimal solution of the original problem for at least one problem $W^{P}$.

For each problem $j \in \mathrm{W}^{p}$ either, although getting closer $\Gamma\left(x^{j}\right)$, does not coincide with $\phi\left(x^{p}\right)$, or $\Gamma\left(x^{j}\right)>v$. This is a condition ensuring that some progress towards the final solution is made. The combinatorial tree has each node identified with a subproblem j. The problems that replace $\mathrm{j}$ in the bounding set $\mathrm{W}^{p}$ are pointed to by the branches directed outward from that node. At any intermediate point in the calculations, the set $W^{p}$ of the current bounding problems is identified with the set of nodes that are the leaves of the tree. Any leaf node of the tree whose bound is strictly less than $y$ is active. Otherwise it is designated as terminated and need not be considered in any further computation. The B\&B tree will be developed until every leaf can be terminated.

\section{Overall probability of failure with respect to plastic collapse}

\section{First and second-order bounds}

According to Equation 31, the probability of failure of a ductile structure may be evaluated as the probability of 
the union of failure mode events. Collapse modes are usually correlated through loading and resistances, so an exact evaluation of the probability is impractical, or even impossible to perform numerically. For this reason, several investigators considered this problem by either finding bounds for $p_{\mathrm{F}}$ or approximate solutions. In general, the admissible failure probability for structural design is very low. A first estimate of $P_{\mathrm{f}}$ can be found through wellknown first-order bounds proposed by Cornell ${ }^{11}$ :

$$
\underset{\text { all } \mathrm{k}}{\operatorname{Max}}\left[\operatorname{Pr}\left(\mathrm{Z}_{\mathrm{k}}\right)\right] \leqslant p_{\mathrm{F}} \leqslant \sum_{\mathrm{k}=1, \mathrm{~m}} \operatorname{Pr}\left[\mathrm{Z}_{\mathrm{k}} \leqslant 0\right]
$$

The lower bound, which represents the probability of occurrence of the most critical mode (dominant mode) is obtained by assuming the mode failure events $Z_{\mathrm{k}}$ to be perfectly dependent, and the upper bound is derived by assuming independence between mode failure events. Hence, approximation by Cornell's first-order upper bound is very conservative because it neglects the high correlation between failure modes in 2-D structures. Improved bounds can be obtained by taking into account the probabilities of joint failure events such as $\mathrm{P}\left(\mathrm{F}_{i} \cap \mathrm{F}_{j}\right)$ which means the probability that both events $F_{i}$ and $F_{i}$ will simultaneously occur. The resulting closedform solutions for the lower and upper bounds are as follows:

$$
\begin{aligned}
& p_{F} \geqslant \mathrm{P}\left(\mathrm{F}_{1}\right)+\sum_{i=2}^{\mathrm{m}} \operatorname{Max}\left\{\left[\mathrm{P}\left(\mathrm{F}_{i}\right)-\sum_{j=1}^{i-1} \mathrm{P}\left(\mathrm{F}_{i} \cap \mathrm{F}_{j}\right)\right] ; 0\right\} \\
& p_{\mathrm{F}} \leqslant \sum_{i=1}^{\mathrm{m}} \mathrm{P}\left(\mathrm{F}_{i}\right)-\sum_{i=2}^{\mathrm{m}} \underset{j<i}{\operatorname{Max}} \mathrm{P}\left(\mathrm{F}_{i} \cap \mathrm{F}_{j}\right)
\end{aligned}
$$

The above bounds can be further approximated using Ditlevsen's $\mathrm{s}^{12}$ method of conditional bounding to find the probabilities of the joint events. This is accomplished by using a Gaussian distribution space in which it is always possible to determine three numbers $\beta_{i}, \beta_{j}$ and the correlation coefficient $\rho_{i j}$ for each pair of collapse modes $\mathrm{F}_{i}$ and $\mathrm{F}_{j}$.

Improved bounds can also be obtained by using Vanmarcke's ${ }^{13}$ concept of failure mode decomposition which takes into account the conditional probability that the $(i-1)$ mode survives given that mode $i$ occurs. By assuming that the probability of occurrence of the $i$ th mode $\mathrm{P}\left(\mathrm{F}_{i}\right)=\Phi\left(\beta_{i}\right)$ depends on $\beta_{i}$ only, the conditional probability $\mathbf{P}\left(\mathrm{S}_{j} \mid \mathrm{F}_{i}\right)$ is evaluated in terms of the safety indices $\beta_{i}$ and $\beta_{j}$ and of the coefficient of correlation $\rho_{i j}$ between the failure modes $F_{i}$ and $F_{j}$.

A different approximate method which avoids calculating conditional probabilities resulting from conditions leading to failure via pairs of failure modes is the PNET $^{14}$. This method requires the determination of the coefficients of correlation between any two failure modes $i$ and $j$ and is based on the notion of demarcating correlation coefficient $\rho_{0}$ assuming those failure modes with high correlation $\left(\rho_{i, j} \geqslant \rho_{0}\right)$ to be perfectly correlated and those with low correlation $\left(\rho_{i, j}<\rho_{0}\right)$ to be statistically independent. This method is not very convenient for 2-D structures because all the failure modes have a relatively high correlation and the solutions will be heavily dependent on the assumed demarcating coefficient $\rho_{0}$.

Methods which automate the search for other stochastically dominant mechanisms will be described next.

\section{Identification of the stochastically most significant collapse modes}

It is assumed that both load and plastic resistances are perfectly correlated within each finite element. The finite element model can be regarded as a direct extension of the modelling of flexural plane frames. Modal deformations are plastic rotations in the case of frames and the intersection of two (or more) element sides in the case of 3-D structures. A branch and bound tree strategy associated with a strong branching rule is adopted implicitly to enumerate all the dominant modes. The first node of the combinatorial tree corresponds to the stochastically dominant mode. Assuming that the number of finite elements where plasticity develops in most important mode are $r, r$ concave quadratic programs are solved in the first level. The strain parameters associated with each of these finite elements are prevented from entering the basis by setting their value to zero. All modes with a reliability index below a cutoff value are saved. The next step is to consider pairs of finite elements where plasticity develops and all the modes for which a given $\beta_{\max }$ is not exceeded are saved. The procedure is repeated for groups of more than two elements until all combinations are implicitly enumerated.

If the random shape of each yield surface is modelled by associating a resistance random variable to each face of the surface, the number of potential modes of collapse increase and the procedures described in Reference ${ }^{9}$ should be employed.

\section{Numerical example}

The shell supported by beams along its contour represented in Figure 1 will be used to illustrate the procedure.

The geometry of the shell is characterized by its thickness $h$ and by the form of the middle surface shown in Figure 2.

For the global system of coordinates $O X_{1} X_{2} X_{3}$, the middle surface can be defined through the equation,

$$
X_{3}=\mathrm{z}\left[1-\left(X_{1}^{2}-X_{2}^{2}\right) /\left(2 \mathrm{a}^{2}\right)\right]
$$

where $\mathrm{z}$ is the $X_{3}$ coordinate at the centre of the shell and

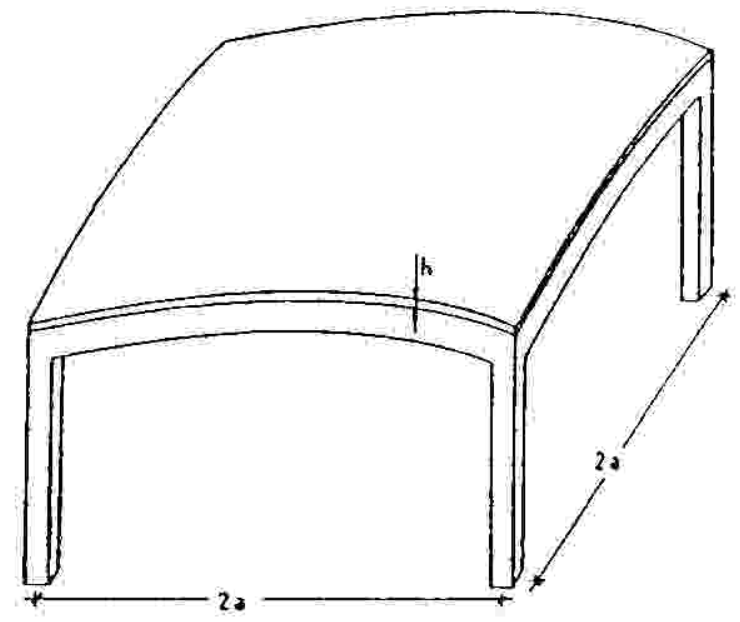

Fig 1 
$2 \mathrm{a}$ is the distance between pairs of columns. The curvatures of the shell are constants given by

$$
\partial_{11} X_{3}=\partial_{22} X_{3}=-z / a^{2} ; \quad \partial_{12} X_{3}=0
$$

The shell degenerates into a square plate if $\mathrm{z}$ is set equal to zero. The shell is discretized into curved triangular finite elements represented in Figure 3 and each finite element is regarded as a shallow curved plate.

The governing relations at finite element level can be derived first from the local coordinate axes referring to each finite element.

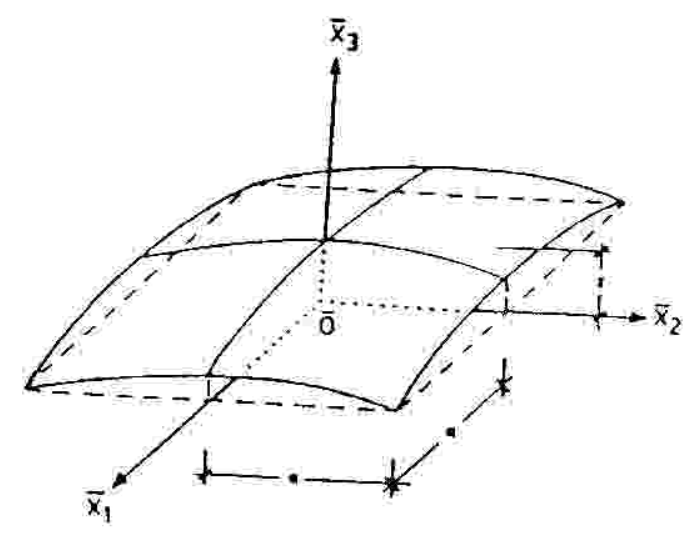

Fig 2

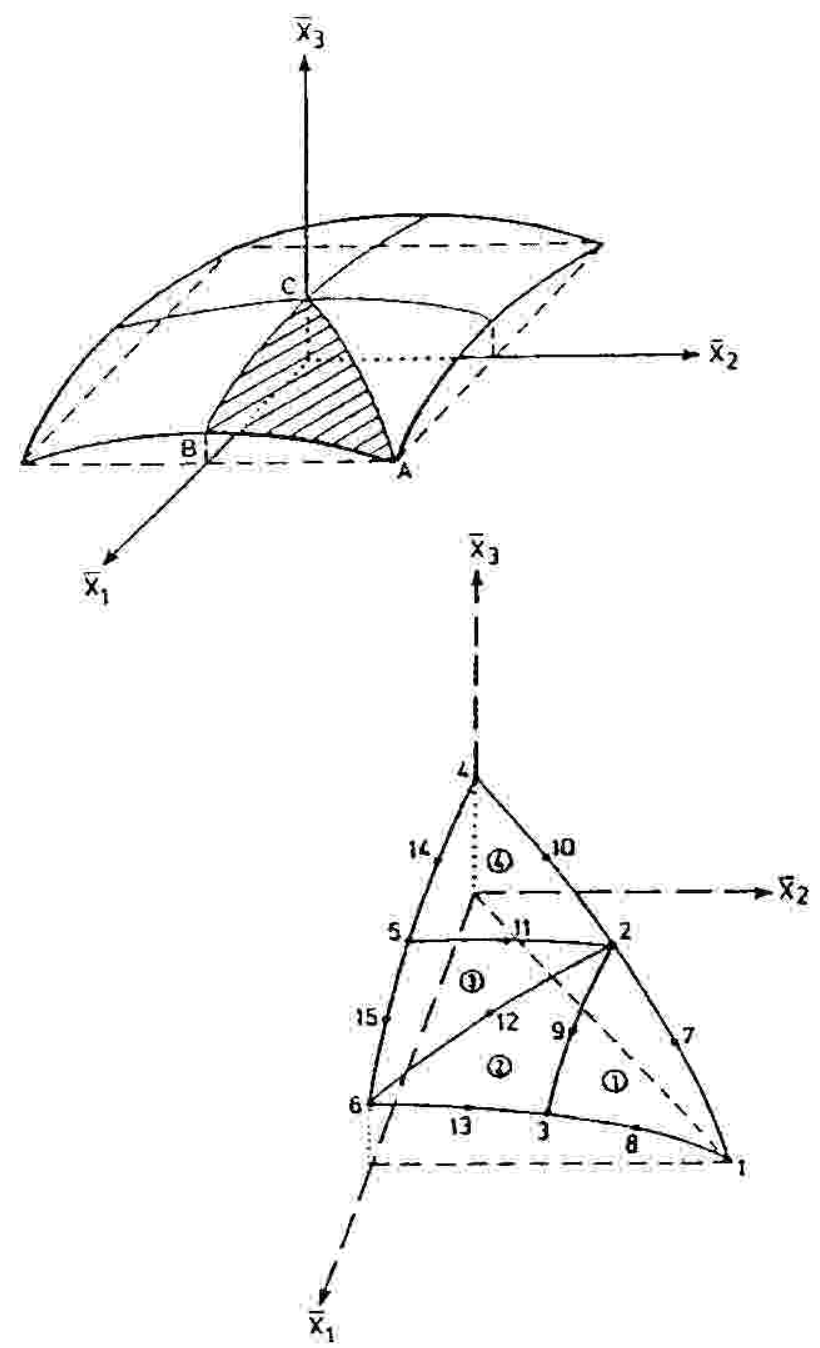

Fig 3 Octant of the shell and finite element discretization

\section{Field functions}

Since stretching and bending problems are individualized in the governing relations of shallow curved plates and since the kinematic model under consideration can be taken as the superposition of stretching and bending models, field functions may be obtained through the superposition of field functions defined for those modes. Three nodal triangular elements are used to model stretching. The displacement field $\Gamma_{u} s$ is assumed linear on the coordinates and the stress field $\Gamma_{\delta} s$ is taken constant inside each finite element. Nodes a are corner nodes. Constant stress field may be defined by the nodal stresses at a single node anywhere inside the finite element. Thus, the single node $\mathrm{e}$ is coincident with the single control node $c$.

Six nodal triangular finite elements are used to model bending. A quadratic deflection field $\Gamma_{u} b$ and thus six nodal displacement values must be specified: vertical deflections at element corners and mid-side normal rotations. A constant moment field $\Gamma_{\sigma} b$ is considered and consequently strain compatibility is fulfilled because no approximation is involved in transformation (Equation 6).

\section{Kinematic transformation matrix $E$}

For thin shells, straight lines normal to the middle surface are assumed to remain normal to the deformed middle surface, that is Kirchhof's hypothesis of negligible shear deformations. The strain vector is similar to that for stretching problems with extra terms accounting for the curved form of the finite element.

For every finite element complying with the assumption of shallow curved plate theory

$$
\begin{aligned}
\mathrm{E} & =\int_{\mathrm{A}} \Gamma_{\sigma}^{t} \mathrm{D} \Gamma_{u} \mathrm{dA} \\
& =\int_{\mathrm{A}}\left|\begin{array}{rr}
\Gamma_{\sigma} s^{t} & \\
& \Gamma_{\sigma} b^{t}
\end{array}\right|\left|\begin{array}{rr}
\mathrm{D}_{s} & -\partial z \\
& \mathrm{D}_{b}
\end{array}\right|\left|\begin{array}{rr}
\Gamma_{u} s & \Gamma_{u} b
\end{array}\right| \mathrm{dA}=\left|\begin{array}{cc}
\mathrm{E}_{s} & \mathrm{E}_{c} \\
0 & \mathrm{E}_{b}
\end{array}\right|
\end{aligned}
$$

where $\partial_{i j} z=\partial^{2} z /\left(\partial X_{i} \partial X_{j}\right)$ are the curvatures of the shell mid-surface with respect to the global coordinate axes $X$. $\mathrm{D}_{s}$ and $\mathrm{D}_{b}$ are matrix differential operators for plate stretching and plate bending problems, respectively. $\mathbf{E}_{s}$ and $\mathrm{E}_{b}$ are the kinematic transformation matrices for stretching and bending problems, respectively. $\mathrm{E}_{c} \mathrm{ac}-$ counts for the curvilinear local axes of the finite element.

\section{Transformation of coordinates}

The variables involved in the formulation are the displacements $u^{a}$ at nodes a (corner and midside nodes) and the total strain-resultants $g^{c}$ at control nodes $c$ (one per finite element at any position inside the element). Nodes e are the mid-side nodes. No change of coordinates is performed at the control nodes and thus transformation matrix $\mathrm{T}_{g}^{c}$ is a unit matrix. The transformation matrix $\mathrm{T}_{u}^{a}$ for displacements $u^{a}$ is easily established in terms of the direction cosines of the local axes with respect to the global system of reference. The direction cosine $\cos B_{i j}$ is given by the following inner product,

$$
\cos B_{i j}=i_{i} j_{j}
$$

where the local and the global axes are represented by $\dot{i}_{i}$ 
and the unit vectors $j_{j}(j=1,2,3)$, respectively. For the present case the transformations of the displacements $u_{1}$, $u_{2}$ and $u_{3}$ at the corner nodes 1,2 and 3 and the normal rotations $\theta_{4}, \theta_{5}$ and $\theta_{6}$ at mid-side nodes 4,5 and 6 are required.

The transformation matrix $T_{s}^{\mathrm{E}}$ is,

$$
\cos \mathrm{B}_{\mathrm{kj}}=\mathrm{n}_{\mathrm{k}} \mathrm{j}_{\mathrm{j}}
$$

with $\mathrm{n}_{\mathrm{k}}$ is the unit vector which is tangent to the shell mid-surface and normal to the element side corresponding to the mid-side node $i(i=4,5,6)$.

\section{Matrix R}

Considering that there is one single control node,

$$
\left.\mathrm{R}^{c}=\mathrm{R}=\left[\mathrm{T}_{\sigma}^{e}\right)^{t}\left(\Gamma_{\sigma}^{c}\right)^{t} \mathrm{~T}_{g}^{c}\right]=\left|\begin{array}{ll}
\left(\mathrm{T}_{\alpha} s^{e}\right)^{t} \Gamma_{\sigma} s^{t} & \\
& \left(\mathrm{~T}_{\sigma} s^{e}\right)^{t} \Gamma_{\sigma} s^{t}
\end{array}\right|
$$

\section{Normal matrix $\mathrm{Q}$}

For simplicity, the yield criterion used here considers separately the stretching and bending problems with no interaction between them. Both for the in-plane forces and for the moments, the Nielsen ${ }^{15}$ yield criterion formulation is adopted. The corresponding simplified yield surface for bending (and stretching) consists of two intersecting cones. Such a yield surface may be linearized as suggested by Wolfenberger ${ }^{16}$ who considers eight planes as represented in Figure 4 for bending. Both for the inplane forces and for the moments, the linearized yield surfaces are octaedrums characterized by the overall normal matrix:

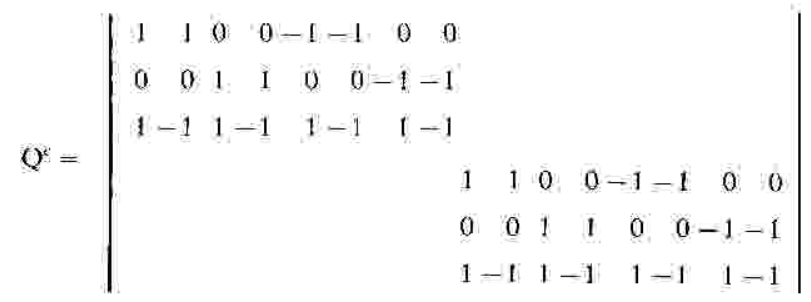

and since there is only one control node inside each finite element,

$$
\mathrm{Q}^{c}=\mathrm{Q} \text {. }
$$

The transformation from local to global coordinates is considered in accordance to

$$
L^{a}=\left(\mathrm{T}_{u}^{a}\right)^{t} \mathrm{~L}^{a}
$$

\section{Random variables}

Two independent random variables associated with loading are considered: Dead $\left(\mu_{1} \mathrm{~d}, \Omega_{1} \mathrm{~d}\right)=\left(5 \mathrm{kN} / \mathrm{m}^{2}\right.$, $0.10)$ and live loading $\left(\mu_{1} 1, \Omega_{1} 1\right)=\left(5 \mathrm{kN} / \mathrm{m}^{2}, 0.10\right)$, the yield surface is described by a single random variable. Each yield surface has then a deterministic shape with a random size. It is further assumed that the plastic resisting capacities are perfectly correlated within finite elements and between them $\left(\mu_{\sigma}, \Omega_{\sigma}\right)=\left(\mu_{\sigma} s, \mu_{\sigma} b, \Omega_{\sigma}\right)$ $=(20000 \mathrm{kN} / \mathrm{m}, 100 \mathrm{kNm} / \mathrm{m}, 0.10)$.

In Figure 5 it is represented by the variation of the reliability index $\beta=\Phi^{-1}\left(1-p_{\mathrm{F}}\right)$ of the stochastically most important mode (for $\mathrm{a}=5 \mathrm{~m}$ ) with respect to the $\mathrm{z} / \mathrm{a}$ ratio. Several coefficients of variation of the plastic capacities are considered.

Figure 6 shows how the reliability index changes with respect to the $2 /$ a ratio. Several coefficients of variation of the live loading are considered. Finite elements 1,2 and 4 become plastic in the stochastically dominant mode. Two alternative mechanisms, corresponding to the development of plasticity in finite elements 1,4 and 2,4 , respectively, are highly correlated with the stochastically dominant mode. The overall probability of failure given by Ditlevsen (upper bound), Ditlevsen (lower bound) or Vanmarcke (upper bound) remains identical to Cornell's lower bound. This example shows that the effect of the plastic resistances is dominant as $z / a$ increases.

If the plastic resistances are $\left(\mu_{\sigma} s, \mu_{\sigma} b\right)=(10760 \mathrm{kN} / \mathrm{m}$, $53.8 \mathrm{kNm} / \mathrm{m}),(4980,24.9),(1790,8.9) .(800,4)$ for $\mathrm{a} / \mathrm{z}$ equal to $0.05,0.1,0.2,0.5$, respectively, the shells would have the same reliability as the square plate with $(20000 \mathrm{kN} / \mathrm{m}, 100 \mathrm{kNm} / \mathrm{m})$.

On the other hand, if the dimension a is $10 \mathrm{~m}$ for $\left(\mu_{\sigma} s, \mu_{\sigma} b\right)=(20000 \mathrm{kN} / \mathrm{m}, 100 \mathrm{kNm} / \mathrm{m})$, the shell with $\mathrm{z} / \mathrm{a}=0.1$ has the same reliability as the square plate with $\mathrm{a}=5 \mathrm{~m}$.

The effect of the finite element discretization on the reliability assessment will be analysed next. If 16 and 4 finite elements are considered per octant of the shell, the following results are obtained:

\begin{tabular}{rlllll} 
& $z / \mathbf{a}=0$ & 0.05 & 0.1 & 0.2 & 0.5 \\
\hline 16 elements & $\beta=2.547\left(p_{\mathrm{F}}=5.4110^{-3}\right)$ & 5.746 & 8.155 & 9.401 & 9.738 \\
4 elements & $\beta=2.396\left(p_{\mathrm{F}}=8.2710^{-3}\right)$ & 5.629 & 8.099 & 9.384 & 9.731
\end{tabular}

\section{Loading vectors}

Concentrated forces are assigned directly to the relevant nodes and distributed forces are substituted by the equivalent nodal forces through integration. The uniformly distributed body force field is accounted for as follows:

$$
\mathrm{L}^{a}=\int_{\mathrm{A}} \Gamma_{u}^{t} b \mathrm{dA}=\int_{\mathrm{A}}\left|\begin{array}{cc}
\Gamma_{u}^{s} & b^{s} \\
\Gamma_{u}^{b} & b^{b}
\end{array}\right| \mathrm{dA}=\left|\begin{array}{c}
\mathrm{L}_{s}^{a} \\
\mathrm{~L}_{b}^{a}
\end{array}\right|
$$

Therefore, the safe linearization of the constitutive relations prevails over the upper bound nature of the kinematic formulation and leads to a conservative estimate of the probability of failure.

The probability of failure changes with the number of random variables and the correlation between them. If it is assumed that the plastic stress capacities are perfectly correlated within the finite element but each element is associated with an independent random capacity, the following results are obtained for $\mathrm{z} / \mathrm{a}=0$ :

$$
\text { Cornell (lb) }
$$

Ditlevsen (b)

Ditlevsen (ub)

f.e. uncorrelated
perfect correlation between t.e.

$2.5510^{-3}$

$8.2710^{-3}$
$2.5910^{-3}$

$8.2710^{-3}$ 


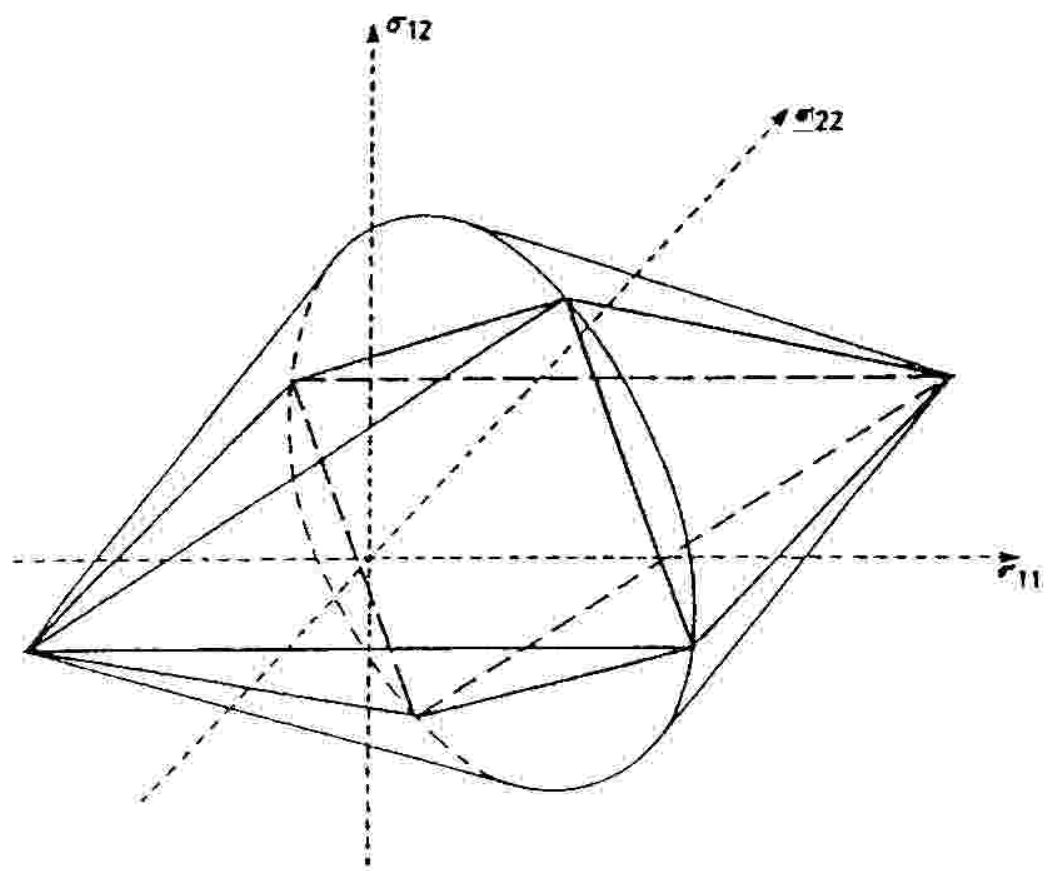

Fig 4

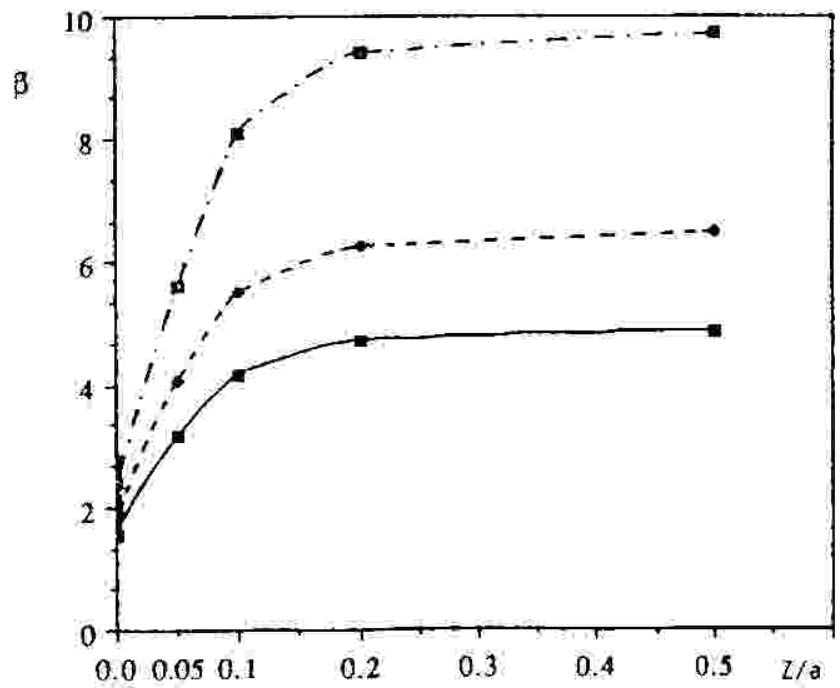

- $\Omega m=0.10-.-$

- $\Omega \mathrm{m}=0.15 \ldots$

Fig 5

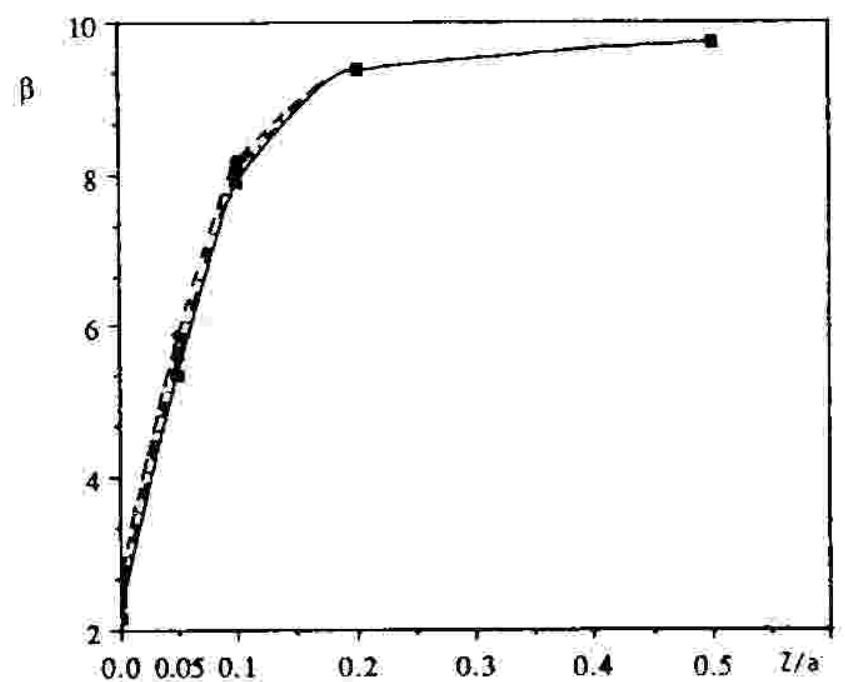

- $\Omega=0.25-$.

- $\Omega=0.20$

- $\Omega \mathrm{l}=0.30$

Fig 6 
The probability of failure increases with increasing correlation between plastic resistances (and between loads). Therefore it is conservative to assume that the plastic are perfectly correlated. Also, an assumption of independent yield surfaces may be very inappropriate and in the unsafe side.

\section{Conclusions}

This paper has been concerned with the development of mathematical programming techniques for the reliability assessment of three-dimensional structures. The finite element method was chosen because of its versatility and it is assumed that plastic collapse is the only possible failure mode. A shallow curved plate element is considered for which it is necessary to define parametric stress and displacement fields. The kinematics and statics are governed by relations where the stretching is dissociated from the bending problem. The kinematic model under consideration can be taken as the superposition of stretching and bending models and the field functions required may be obtained through the superposition of the field functions defined for those models. For simplicity, the yield criterion used here considers separately the stretching and bending problems with no interaction between them. In order to avoid the difficult numerical integration of the probability distribution functions of the random variables involved, the first-order secondmoment approximation is employed. The identification of the reliability index is formulated as a constrained concave quadratic minimization. Numerical results for a shallow shell model are presented.

\section{References}

1 Cyras, A. and Kalanta, S. Optimal design of cylindrical shells by the finite element technique, Mech. Res. Comm., I (125), 1974
2 Hung, N. D, and Ransart, D. Analyse limite de coques de revolution par la mèthode des elements finis, Proc. IASS World Cong. Space Enclosures, P. Fuzio (ed.), Concordia University Montreal. 1976

3 Cyras, A., Karkauskas, R. P. and Atkochyunas, Y. Y. Optimization of rigid-plastic shells by the finite element method, Proc. IASS Conf. Lightweight Shell Space Struct. Normal and Seismic Zones, Raizer, V. (ed.). MIR, 1977

4 Küfner, V. J. and Lee, N. Limit analysis of shallow shells using finite elements and linear programming, Proc. YASS Conf, Lightweight Shell Space Struct. Normal and Seismic Zmes, Raizer, V. (ed.). MIR, 1977

5 Da Fonseca, A. M. A. Plastic analysis and synthesis of plates and shells by mathematical programming, Ph.D. Thesis: Imperial Col lege, London, 1980

6 Shinozuka, M. Basic analysis of structural safety, J. Struct. Division, $A S C E, 109,721-40,1983$

7 Rosenblatt, M. Remarks on a multivariate iransformation, Annals of Math. Stat. 23, 1952

8 Schaible, S. and tbaraki, T. Fractional programming, European Journal of Operations Reseurch, 12, 325-38, 1983

9 Simóes, L. M. C. Reliability-based synthesis of portal frames, in Nato-Asi on Decision Support Systems in Civil Engng. Edinburgh, 1989

10 Falk, J. E. and Soland, R. M. An algorithm for separable nenconvex programming problems, Man. Sei, 15, 551-68, 1969

11 Comell, C. A. Bounds on the Reliability of Structural Systems, $J$. Siruct. Div., ASCE, 93, 171-200, 1967

12 Vanmarcke, E. H. Matrix formulation of reliability analysis and reliability-based design, Comp. and Struct. 3, 757-70, 197I

13 Ditleysen, $\boldsymbol{O}$. Narrow reliability bounds for structural systems, $J$. Siruct. Mech., 7, 453-72, 1979

14 Ang, A. H. S. and Ma, H. F, On the reliability of structural systems, 3rd Ine. Conf. on Structural Safety and Reliability, Throndeim, 1982

15 Nielsen, M.P. Yield conditions for teinforced concrete shells in the membrane state, Proc. IASS Symp. Non-classical Shell Problems. North-Holland, 1030, 1963

16 Wolfensherger, R. Traglast und optimale Bemessung von Platten, Diss. ETH Zürich, 1964 